\title{
APPORT DES TECHNIQUES EXAFS ET APPARENTEES A LA DETERMINATION DE L'ORDRE LOCAL: APPLICATION A LA TRIBOLOGIE
}

J. Lopez, j.C. Le Bossé, G. IIansali

Laboratoire de Tribologie et de Dynamique des Systèmes, CNRS URA 855

Ecole Nationale d'Ingénieurs de St Etienne

58 rue Jean Parot, 42023 St Etienne, France

M. Belin Et J.M. Martin

Laboratoire de Tribologie et de Dynamique des Systèmes, CNRS URA 855

Ecole Centrale de Lyon 36 av. Guy de Collongue, BP 163, 69131 Ecully, France

CONTRIBUTION OF EXAFS-LIKE TECHNIQUES TO TIIE DETERMINATION OF LOCAL ORDER: APPLICATION TO TRIBOLOGY: The determination of local order at atomic scale becomes more and more fundamental for understanding superficial phenomena such as tribology and catalysis. Among a few techniques a vailable in this field, we are only concerned with the EXAFS-like ones (EXAFS, SEXAFS, EXELFS, SEELFS, etc.). The difficulties appearing all along the numerical treatment of such spectra are carefully analysed and a methodology is proposed in view to obtain reliable radial distribution functions. Some applications of these techniques are presented in two fields connected with tribology: friction induced amorphisation in high vacuum and characterization of interfacial solid bodies created during a wear process including an antiwear additive.

PACS numbers: $61.10 . \mathrm{Lx}$

\section{Introduction: lien entre tribologie et ordre local}

\subsection{Généralilés}

Bien que les propriétés mécaniques et tribochimiques de corps en frottement aicnt été étudiées depuis fort longtemps, on constate un manque de connaissances en cc qui concerne la corrélation entre la microstructure géométrique et chimique de la zone interfaciale et les propriétés mécaniques qui en découlent. La raison en est simple à comprendre: les méthodes physiques qui permettent d'appréhender 
ces problèmes sont peu nombreuses, difficiles à mettre en oeuvre et souvent d'un développement récent. Pourtant la connaissance du désordre structural induit par frottement, ainsi que des modifications chimiques des corps en présence est fondamentale pour comprendre les propriétés du frottement. Par exemple, dans le cas du frottement sec sous ultra-vide où le désordre ne peut être que structural, l'étude de l'ordre local aura pour but de définir la localisation du cisaillement dans l'interface et le mode de glissement. Deux cas limites sont alors envisageables: si l'on a affaire à des bandes de glissement entre lesquelles très peu de matière est concernée par le frottement, en moyenne l'ordre local n'est pratiquement pas modifié, à l'opposé, s'il se développe une zone interfaciale amorphe constituée de micrograins roulant les uns sur les autres, l'ordre local se trouve fortement altéré (un exemple de ce type sera développé dans Sec. 3.1). Dans un autre domaine, celui de la lubrification limite, c'est l'inférence de la cinétique chimique, en général une oxydation, dans les modifications structurales qui est l'objet de préoccupations (un exemple de ce type sera aussi développé dans Sec. 3.2).

\subsection{Comment alleindre l'ordre local à l'échelle atomique?}

Parmi les rares méthodes permettant d'appréhender l'ordre local à l'échelle atomique: EXAFS (Extended X-Ray Absorption Fine Structure) et méthodes dérivées $[1,2]$, microscopie à effet tunnel, méthodes liées à la diffraction des électrons rapides [3] ou à la diffusion hors des directions de diffraction d'électrons lents [4], ce sont les premières qui sont de loin les plus utilisées et qui seront développées dans cet article. En effet, l'EXAFS comme l'EXELFS (Extended Energy-Loss Fine Structure) sont particulièrement bien adaptées à l'étude des fragments d'usure, tandis que le SEXAFS (Surface Extended X-Ray Absorption Fine Structure) et le SEELFS (Surface Electron Energy-Loss Fine Structure) permettent d'appréhender l'étude des surfaces usées.

A l'aide de ces techniques, toutes liées à l'éjection d'un électron de coeur depuis un atome particulier, on peut espérer atteindre un certain nombre de renseignements capitaux, comme la nature chimique des atomes entourant l'émetteur, le nombre de coordination de chaque espèce chimique entourant l'émetteur et les distances entre l'émetteur et ses voisins. Une autre technique: le XANES (X-Ray Absorption Near Edge Structure), qui s'appuie sur le même type d'expérimentation, mais s'intéresse au seuil d'absorption lui-même plutôt qu'aux structures fines qui le suivent, permet éventuellement de compléter les informations obtenues par les techniques précédentes par de nouvelles informations, tout aussi capitales, sur le degré d'oxydation de l'atome émetteur, la symétrie de son environnement local et parfois la densité d'état partielle des états inoccupés. Bien qu'elle ne soit pas à proprement parler apparentée à l'EXAFS, cette technique sera quand même illustrée par un exemple dans Sec. 3.3 compte tenu de son intérêt.

Si la plupart de ces techniques sont difficiles à mettre en oeuvre, particulièrement celles qui utilisent un rayonnement synchrotron comme l'EXAFS et le SEXAFS, elles présentent l'immense avantage de s'appuyer sur une théorie simple à mettre en oeuvre. Malheureusement, le dépouillement des spectres EXAFS et apparentés présente de nombreuses difficultés qu'il n'est pas toujours facile 
d'éviter. Après avoir rappelé les grandes lignes des bases théoriques de l'EXAFS dans Sec. 2.1, nous nous attacherons dans Sec. 2.2 à mettre en évidence les difficultés apparaissant au cours du traitement des spectres, en les illustrant dans le cas d'un spectre EXELFS correspondant à du carbure de silicium cristallisé. Ce paragraphe aura pour objectif de dégager une méthodologie permettant de garantir la fiabilité des résultats obtenus. Dans Sec. 3 nous présenterons quelques applications marquantes de ces techniques à la tribologie. Une courte conclusion viendra ensuite clore ce travail.

\section{Le traitement des spectre de type EXAFS}

\subsection{Rappels sur l'EXAFS et les méthodes apparentées}

L'EXAFS consiste à faire traverser un échantillon par un faisceau de photons $\mathrm{X}$ d'énergie variable $h \nu$ et à déterminer le coefficient d'absorption $\mu$ des photons déduit de la loi de Lambert

$$
I(h \nu)=I_{0}(h \nu) \mathrm{e}^{-\mu(h \nu) d},
$$

où $I_{0}$ est l'intensité du faisceau incident et $I$ - celle du faisceau transmis. En fait, c'est le rapport

$$
\chi(k)=\frac{\mu-\mu_{0}}{\mu_{0}},
$$

où $\mu_{0}$ serait le coefficient d'absorption de l'émetteur isolé de son environnement et $k$ - le vecteur d'onde de l'électron émis, qui fait l'objet des études EXAFS. $\chi(k)$ isole donc les structures fines, de nature oscillante, qui viennent se superposer $\grave{a} \mu_{0}$. L'origine de ces oscillations est facile à comprendre et est illustrée sur Fig. 1. L'électron éjecté par l'atome absorbeur est assimilable à une onde sphérique libre sortante. Cette onde sortante se réfléchit partiellement sur les atomes entourant l'émetteur. Ceux-ci renvoient alors sur l'atome central un ensemble d'ondes rétrodiffusées qui interférent avec celle qui leur a donné naissance. C'est de ce phénomène d'interférence que naissent les oscillations caractéristiques de l'EXAFS.

Dans la mesure où l'on ne prend en compte que les processus de rétrodiffusion simple dans l'analyse théorique du phénomène, on aboutit à une relation particulièrement facile à utiliser, qui est rappelée ici dans le cas d'un seuil $K$ et d'une lumière non polarisée $[1,2]$ :

$$
\chi(k)=\sum_{j} \frac{N_{j}}{k R_{j}^{2}} f_{j}(k) \sin \left(2 k R_{j}+\phi_{j}(k)\right) \mathrm{e}^{-2 \sigma_{j}^{2} k^{2}} \mathrm{e}^{-2 R_{j} / \lambda(k)}
$$

avec $k$ - vecteur d'onde de l'électron émis, $j$ - indice différenciant les atomes par couche et par nature chimique, $R_{j}$ - distance entre l'atome central et ses voisins de type $j, N_{j}$ - nombre d'atomes de type $j$ situés à la distance $R_{j}, \sigma_{j}-$ coefficient de Debye-Waller des atomes de type $j, \lambda(k)$ - libre parcours moyen du photoélectron, $f_{j}(k)$ - amplitude de rétrodiffusion des atomes de type $j, \phi_{j}(k)-$ déphasage total impliquant un rétrodiffuseur de type $j, \exp \left(-2 \sigma_{j}^{2} k^{2}\right)-$ facteur de Debye-Waller, $\exp \left(-2 R_{j} / \lambda(k)\right)-$ facteur provenant des pertes inélastiques. 

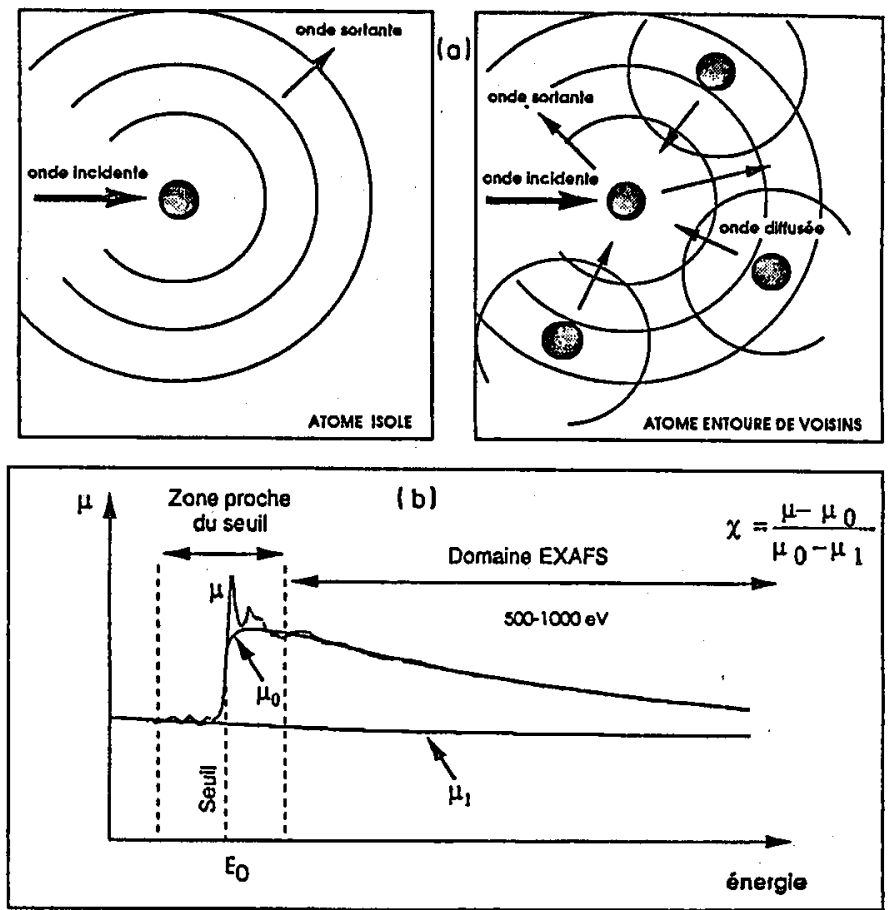

Fig. 1. Représentation simplifiée des processus physiques (a) qui donnent naissance aux oscillations caractéristiques des spectres de type EXAFS et (b) allure de ce type de spectre.

L'analyse directe du faisceau transmis n'est que l'un des différents modes de mesure possible pour l'EXAFS. On peut aussi procéder en étudiant les effets produits par l'absorption des photons comme le rayonnement fluorescent ou l'émission d'électrons secondaires ou Auger. Le SEXAFS est rigoureusement de même nature que l'EXAFS. On se contente simplement de travailler sous incidence rasante afin d'être surtout sensible aux propriétés de surface. L'EXELFS se différencie de l'EXAFS par la nature des particules excitatrices qui sont des électrons au lieu d'être des photons. Bien que la nature de l'excitation reste la même, le phénomène est plus compliqué puisque particules excitatrices et particules émises sont identiques. Les mesures, issues généralement d'un microscope à transmission, sont réalisées de façon légèrement différente. Cette fois, l'énergie du faisceau incident est maintenue constante et on analyse les pertes dans le faisceau transmis. On montre alors que dans la plupart des cas, la formule fondamentale de l'EXAFS reste valable pour l'EXELFS, parce que l'énergie du faisceau incident est importante (couramment de l'ordre de 100 à $200 \mathrm{keV}$ ). Quant au SEELFS, qui reprend l'idée du SEXAFS en conservant une excitation électronique, il utilise des énergies incidentes beaucoup moins élevées qu'en EXELFS (quelques keV seulement), ce qui le cantonne à l'étude de seuils peu profonds souvent $L$ ou $M$ pour 
lesquels les phénomènes de superposition de seuils rapprochés posent de nombreux problèmes. En outre, l'usage de la formule fondamentale de l'EXAFS, justifiée en EXELFS à cause de l'énergie incidente élevée, s'avère discutable en SEELFS. Toutefois des travaux récents tendent à le justifier [5]. Pour toutes ces raisons le SEELFS reste une technique d'appoint moins utilisée, mais qui mériterait de l'être dans certains cas favorables.

L'existence d'une théorie, relativement simple, est un atout important en faveur des techniques du type EXAFS. Pourtant la comparaison expérience-théorie n'est pas aisée à réaliser. En effet, certains ingrédients de la formule fondamentale de l'EXAFS comme les déphasages $\phi_{j}(k)$ ou les amplitudes de rétrodiffusion atomique $f_{j}(k)$ ne sont pas faciles à calculer de façon $a b$ initio. Heureusement, il existe une manière de contourner cette difficulté. En effet, on montre, sur la base de la formule fondamentale de l'EXAFS, que le module de la transformée de Fourier (TF) de $k \chi(k)$ présente des pics centrés sur les distances entre l'atome émetteur et ses voisins, à une correction de déphasage près qui reste à évaluer [6-8]. Le calcul de ce module, appelé distribution radiale, est donc la voie la plus couramment utilisée pour exploiter les spectres de type EXAFS. C'est d'ailleurs celle dont nous discuterons par la suite.

Précisons un peu la propriété précédente, $k \chi(k)$ peut aussi s'écrire

$$
k \chi(k)=\sum_{j} g_{j}(k) \chi_{0 j}(k)
$$

avec

$$
\begin{aligned}
& k \chi_{0 j}(k)=\frac{N_{j}}{R_{j}^{2}} \sin \left(2 k R_{j}+\phi_{j}(k)\right) \mathrm{e}^{-2 \sigma_{j}^{2} k^{2}}, \\
& g_{j}(k)=f_{j}(k) \mathrm{e}^{-2 R_{j} / \lambda(k)} .
\end{aligned}
$$

La TF associée à la $j$-ème couche se met alors sous la forme

$$
k \chi_{0 j}(k) g_{j}(k) \Leftrightarrow F_{j}(R) * G_{j}(R)
$$

où les symboles $\Leftrightarrow$ et $*$ représentent respectivement la TF et le produit de convolution. $F_{j}(R)$ et $G_{j}(R)$ sont évidemment les TF de $k \chi_{0 j}(k)$ et de $g_{j}(k)$. Dans la mesure où l'on suppose que la phase varie linéairement avec $k$ :

$$
\phi_{j}(k)=-2 k R_{0 j}+\phi_{0 j} \text {, }
$$

le calcul de $F_{j}(R)$ se fait algébriquement. La propriété précédente est d'ailleurs bien vérifiée pour les éléments légers. Le module de la fonction obtenue présente alors un pic centré sur la position $R_{j}^{*}=R_{j}-R_{0 j}$, où $R_{0 j}$ est la fameuse correction de déphasage. La convolution de $F_{j}(R)$ par la fonction $G_{j}(R)$, qu'on ne peut éviter, vient perturber le résultat précédent. On espère simplement que la position du pic n'est pas altérée par cette convolution, ce qui n'est vrai que si la fonction $g_{j}(k)$, qui ressemble en général à une courbe en cloche, est symétrique par rapport à l'abscisse de son sommet. Du fait de cette convolution, le facteur de pondération théorique $k$ de $\chi(k)$ est rarement utilisé. On lui préfère un facteur du type $k^{n}$ ou le degré $n$ est généralement choisi égal à 3 , ce qui modifie la fonction de convolution de toute façon inconnue. Signalons pour finir que la superposition des TF des diverses couches se traduit par un phénomène d'interférence entre les contributions de chaque couche susceptible d'altérer la position des pics. Par conséquent, 
l'analyse traditionnelle de la distribution radiale n'a de sens que pour des pics bien individualisés, condition qui n'est en général remplie que pour la première couche ou dans les cas favorables pour les toutes premières couches.

\subsection{Analyse des problèmes liés au trailement des spectres}

\subsubsection{Introduclion}

En principe, le traitement des spectres de type EXAFS est simple. En pratique, il présente souvent de nombreuses difficultés. C'est le cas des spectres liés à la tribologie parce que: complexe,

- la composition chimique (et/ou géométrique) des zones de frottement est

- on souhaite utiliser des seuils peu profonds ( $L$ ou $M$ ) plus sensibles et/ou plus sélectifs pour la zone de frottement, le faible nombre des émetteurs introduit un bruit important dans les spectres,

- la zone d'analyse est souvent limitée en amplitude (200 à $300 \mathrm{eV}$, alors qu'elle est de l'ordre de $1000 \mathrm{eV}$ en EXAFS traditionnel).

Le traitement des spectres EXAFS ou apparentés liés à la tribologie s'avère donc une opération délicate si l'on veut éviter de nombreuses difficultés comme l'apparition de pics fantômes dans la distribution radiale, d'origine purement numérique, ou des positions de pics qui dépendent étroitement du traitement numérique subi par les spectres.

Afin de mettre en évidence les difficultés liées au traitement des spectres de type EXAFS et de proposer une méthodologie pour s'en affranchir, nous allons procéder à un certain nombre d'essais numériques sur un spectre EXELFS de $\beta$-SiC cristallisé correspondant au seuil $K$.

\subsubsection{Déterminalion de $\chi$}

La première étape du traitement d'un spectre consiste à extrapoler la ligne de base précédent le seuil sous ce dernier, puis à soustraire la courbe obtenue du spectre de départ afin d'en éliminer la partie correspondant à la queue des seuils antérieurs ou des phénomènes qui précèdent le seuil étudié. Les deux principaux modèles utilisés pour cette ligne de base sont la loi de Victoreen [9], issue de la théorie de l'absorption des photons par la matière, ou tout simplement la droite. Figure $2 b$ donne un exemple utilisant la loi de Victoreen. Cette étape conditionne une évaluation ultérieure correcte de $\mu_{0}$ donc de $\chi$. Ce point n'a en fait d'importance que si l'on souhaite effectivement utiliser la formule fondamentale de l'EXAFS. Dans la mesure où l'on opère par TF, comme la courbe soustraite est à variation très lente, l'influence sur l'analyse radiale est faible. Une erreur au cours de cette étape ayant peu d'importance lorsque l'objectif visé est un calcul de distribution radiale, par la suite nous utiliserons donc un modèle linéaire pour la ligne de base. D'ailleurs, la loi de Victoreen n'est pas justifiée en spectroscopie d'électrons.

Il faut ensuite éliminer le fond sur lequel s'appuient les oscillations pour obtenir $\chi$. Il s'agit là d'une phase cruciale, car mal menée elle est susceptible d'introduire des pics parasites dans la distribution radiale du côté des faibles distances. Différentes méthodes sont envisageables: moindres carrés polynomiaux, 


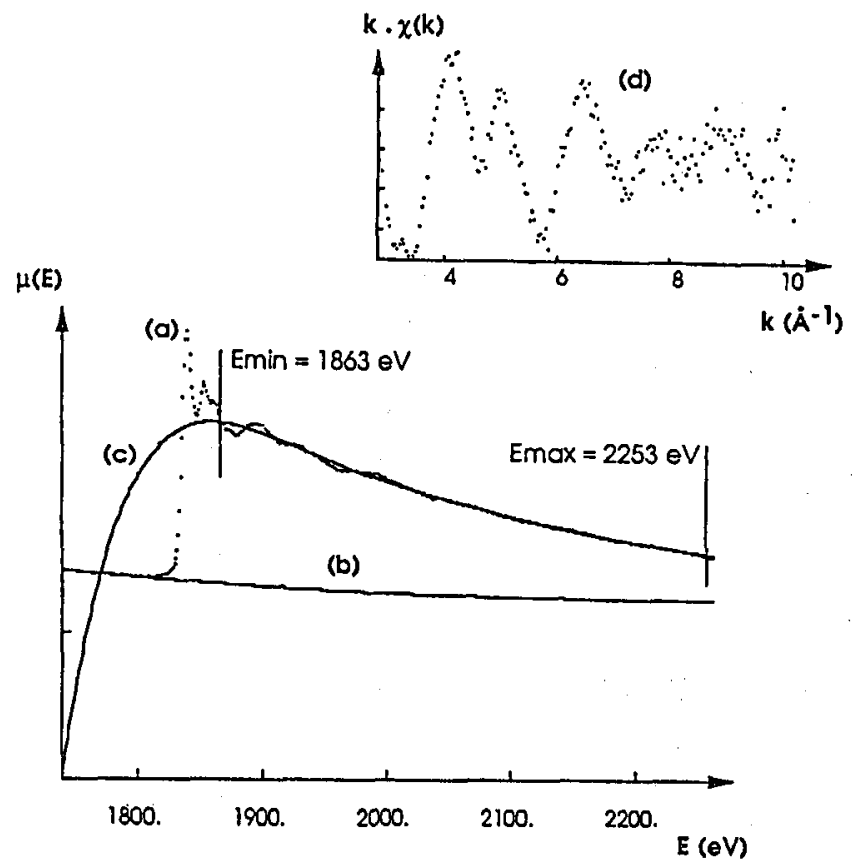

Fig. 2. Cette figure montre: (a) Le spectre EXELFS du seuil $K$ du silicium dans $\beta$-SiC cristallisé. (b) Un exemple d'extrapolation de la ligne de base précédent le seuil $\mu_{1}$ sous celui-ci. La fonction d'extrapolation est du type Victoreen, c'est-à-dire $a+b / E^{3}+c / E^{4}$. Les paramètres $a, b$ et $c$ sont déterminés par la méthode des moindres carrés. (c) Un excmple de lissage des oscillations par la méthode des moindres carrés appliquée entre les deux barres indiquées sur la figure. Le degré du polynôme de lissage est 6. (d) La quantité $k\left(\mu-\mu_{0}\right) /\left(\mu_{0}-\mu_{1}\right)$ qui sert de point de départ à toute analyse de type EXAFS.

moyenne glissante itérée, lissage à base de fonctions splines cubiques, ... Pour des raisons de simplicité, notre discussion portera uniquement sur la première méthode proposée. En fait, les grandes caractéristiques des comportements observés sont les mêmes pour toutes les méthodes. La discussion qui suit a donc une portée générale. Figure 2c donne un exemple d'estimation de fond à l'aide d'un polynôme de degré 6 .

Lorsqu'on augmente le degré du polynôme de lissage, on passe progressivement de la droite des moindres carrés au polynôme d'interpolation. Si le degré est trop faible, le fond réel est susceptible d'osciller autour du fond calculé. Ces oscillations introduisent alors des pics parasites pour les petites distances (Fig. 3), qui interfèrent d'autant plus avec la distribution radiale cherchée que la zone d'analyse est peu étendue (cas de l'EXELFS). Lorsqu'on augmente le degré du polynôme de lissage, on permet à celui-ci d'osciller de plus en plus. On arrive alors à éliminer complètement les pics parasites précédents de la distribution radiale. Malheureusement, le spectre fréquentiel du fond obtenu recouvre de plus en plus 


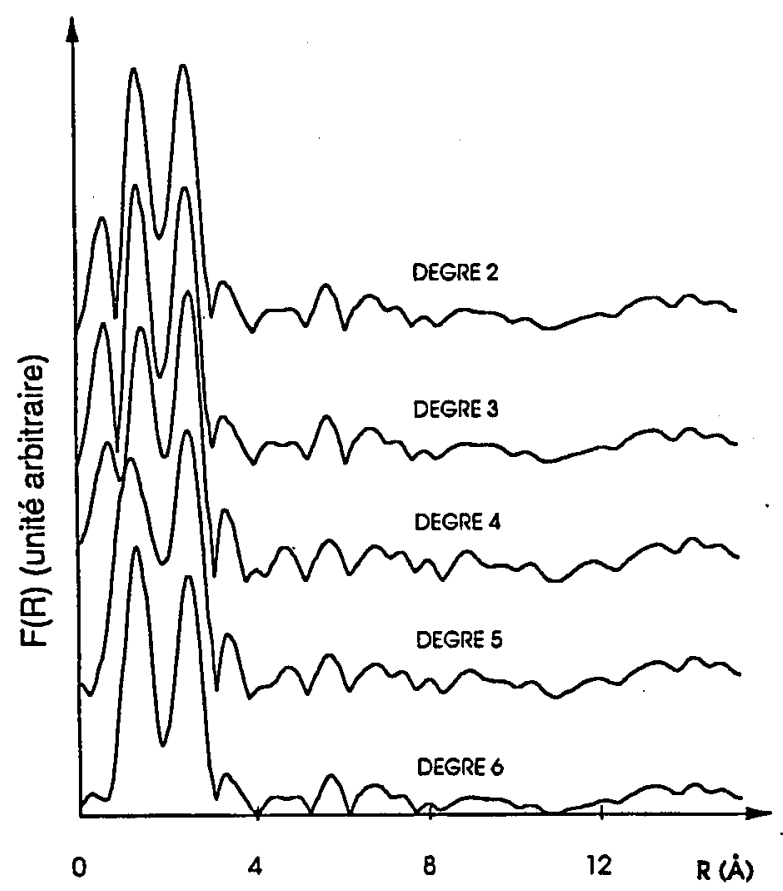

Fig. 3. Cette figure montre l'influence du degré du polynôme de lissage sur la distribution radiale finale $F(R)$. Tous les autres paramètres sont fixés aux valeurs de référence indiquées dans l'annexe.

celui de la distribution radiale. Il en découle une altération de celle-ci qui se traduit d'abord par une modification de l'amplitude du premier pic suivie éventuellement d'une dérive de sa position. Il s'agit évidemment là d'effets indésirables qu'il faut maîtriser. Sur Fig. 3, on observe par exemple un décalage des premiers pics significatifs dû à la présence d'un pic parasite important sur leur gauche pour les faibles degrés. Pour les degrés plus élevés, la position des pics se stabilise, par contre leur amplitude se modifie progressivement. En conclusion, il vaut parfois mieux conserver des petits pics parasites surtout s'ils sont bien résolus, plutôt que de les éliminer en altérant amplitude et position des premiers pics significatifs.

Signalons pour finir que le choix du domaine dans lequel les moindres carrés sont calculés n'est pas neutre. Du côté des grands vecteurs d'ondes, le choix de la coupure est peu important parce qu'on peut espérer que le bruit est équilibré (sauf si l'on observe l'émergence d'un nouveau seuil). Par contre, ce n'est pas le cas du côté des petits vecteurs d'ondes où les toutes premières oscillations, régies par la diffusion multiple, sont rarement équilibrées. Le choix d'une coupure du spectre trop basse se traduit souvent par l'introduction de pics parasites du côté des petites distances dans la distribution radiale. Cet effet est illustré sur Fig. 4. 

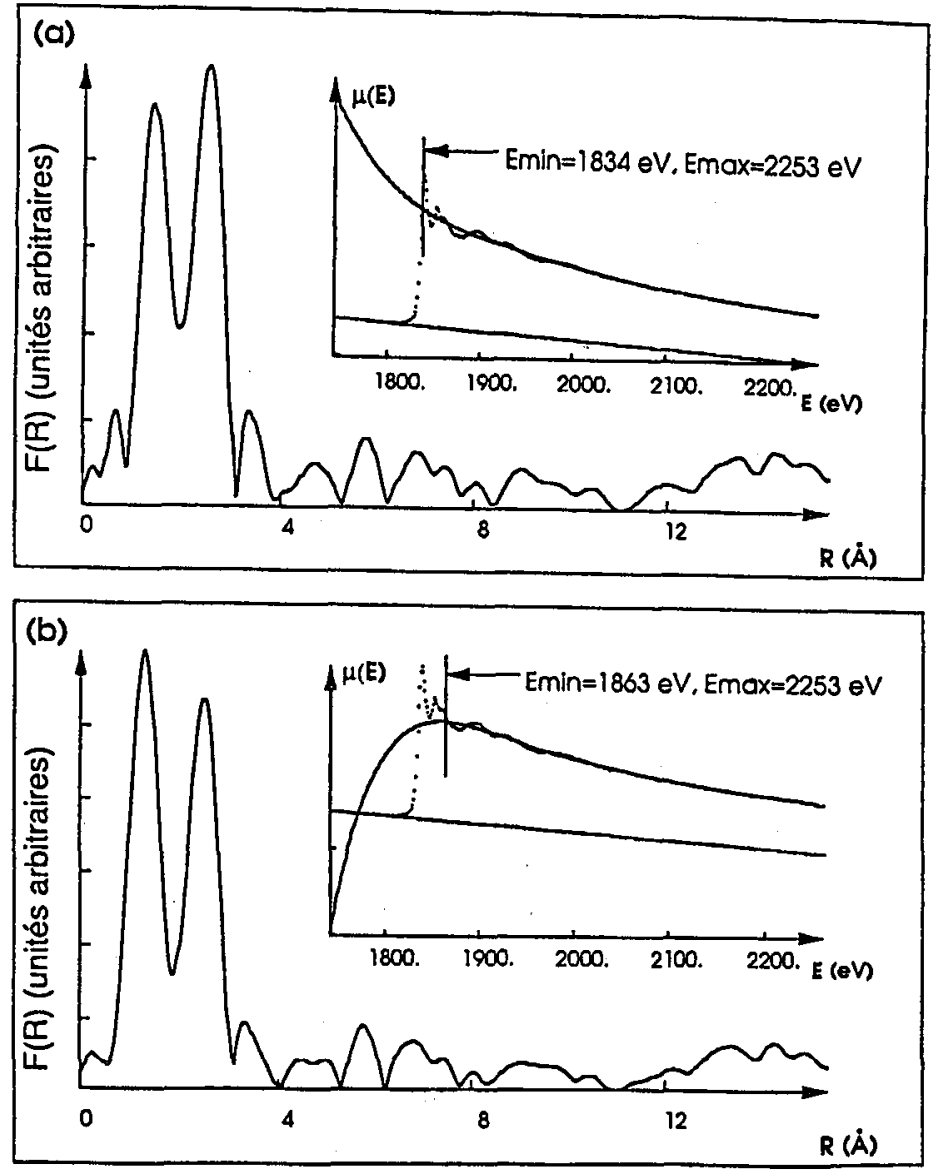

Fig. 4. Cette figure montre l'influence du choix des bornes du domaine de lissage sur la distribution radiale finale: (a) distribution radiale obtenue en évaluant $\mu_{0}$ dans un domaine incluant la première oscillation suivant le seuil, (b) distribution radiale de référence.

\subsubsection{Choix de l'énergie de seuil $E_{0}$}

Il faut ensuite fixer la position du seuil d'absorption $E_{0}$, ce qui n'est pas toujours aisé, et passer de la variable énergie à la variable vecteur d'onde. Le vecteur d'onde étant défini par la relation

$$
k=\sqrt{\frac{2 m}{\hbar^{2}}\left(E-E_{0}\right)}
$$

un mauvais choix de l'énergie de seuil $E_{0}$ a pour effet de distordre la distribution des vecteurs d'ondes, surtout du côté des petites valeurs. L'évaluation de la sensibilité des résultats finaux à une telle distorsion entre dans la caractérisation de la précision finale. Dans le cas considéré les calculs montrent que la distribution radiale est très peu sensible au choix de $E_{0}$, sauf pour les couches éloignées. 
Cela permet de s'affranchir de l'étude du rôle de ce paramètre, mais ce n'est pas toujours le cas.

\subsubsection{Choix de la fenêtre d'analyse}

Avant de calculer la TF, il faut choisir le domaine d'intégration. On a évidemment intérêt à choisir un domaine d'intégration le plus grand possible pour améliorer la résolution. Toutefois deux écueils sont à éviter. Du côté des petits vecteurs d'ondes la formule fondamentale de l'EXAFS n'est pas valide, car les phénomènes de diffusion multiple sont importants. Il faut donc éliminer cette zone. Du côté des grands vecteurs d'ondes, le bruit, amplifié par le facteur de pondération $k$ ou $k^{3}$, remplace progressivement le signal significatif. Il faut donc aussi éliminer celte zone. Dans le cas qui nous intéresse, l'inclusion de la zone qui suit immédiatement le seuil dans le calcul de la distribution radiale n'introduit aucune perturbation significative. Cela semble indiquer une faible contribution des phénomènes de diffusion multiple. Cette propriété a déjà été observée lors d'études EXAFS [10]. Elle est probablement due au caractère ouvert de la structure du SiC. Il ne faut donc pas en conclure hâtivement que ce résultat est général. La troncature de la fin du spectre n'introduit pas non plus de distorsion significative de la distribution radiale, ce qui signifie que le domaine des mesures utilisé n'est pas suffisamment étendu pour que l'amplification du bruit par le facteur de pondération vienne perturber l'analyse radiale.

Le calcul direct de la distribution radiale sur le domaine d'intégration retenu sans pondération particulière est susceptible à son tour d'introduire des pics parasites. Ceci est dû à la mauvaise réponse fréquentielle de la fenêtre naturelle qui présente de nombreux lobes secondaires (par contre elle n'a pas tendance à déplacer les pics). Pour évitcr cet effet, on a donc coutume de pondérer $\chi(k)$ par une fenêtre plus molle que la fenêtre naturelle. A-vrai-dire, de nombreuses fenêtres de pondération sont disponibles (IIamming, IIanning, Gaussienne, Kaiser...). Dans nos calculs nous avons retenu celle de Kaiser qui ne contient qu'un seul paramètre permettant de passer de façon continue de la fenêtre naturelle à une fenêtre d'allure gaussienne. Cette fenêtre est définie par la relation

$$
f(x)=\frac{J_{0}\left(\beta \sqrt{1-x^{2}}\right)}{J_{0}(\beta)} f_{0}(x),
$$

dans laquelle $J_{0}(x)$ est la fonction de Bessel modifiée d'ordre $0, f_{0}(x)$ - la fenêtre naturelle qui vaut 1 si $|x|<1$ et 0 sinon, et $\beta$ est le paramètre de forme. Pour $\beta=0$ on retrouve la fenêtre naturelle.

L'effet d'une variation de la forme de la fenêtre est minime dans le cas du spectre étudié. Seul le petit pic parasite apparaissant sur le pied gauche du premicr pic de la distribution radiale dispara ît progressivement lorsqu'on amollit la forme de la fenêtre. Comme cette supposée amélioration provient essentiellement d'une perte de résolution importante, il vaut mieux s'accommoder des inconvénients d'une fenêtre proche de la fenêtre naturelle. On aboutit d'ailleurs souvent à cette constatation quelle que soit la fenêtre de pondération utilisée.

\subsubsection{Choix du facteur de pondération}

On remplace souvent le facteur de pondération théorique $k$ par le facteur 
$k^{3}$ afin d'amplifier le rôle dcs oscillations en fin de spectre (cf. Sec. 2.2). Dans le cas considéré les deux pics principaux sont peu sensibles à ce choix, surtout en ce qui concerne leur position. Cela justifie, au moins dans ce cas le caractère un peu arbitraire du choix du facteur de pondération. Par contre, pour les couches plus éloignées, les deux distributions radiales diffèrent notablement.

\subsubsection{Conclusion}

La conclusion de cette étude est simple: la seule manière de garantir la fiabilité des résultats obtenus à la suite du traitement d'un spectre de type EXAFS, ainsi que leur précision, consiste à étudier soigneusement le comportement de ce traitement lorsqu'on fait varier tous les paramètres qu'il implique. Dans le cas du spectre étudié on constate une bonne stabilité des résultats dans presque tout le domaine de variation des paramètres. Il s'agit évidemment du résultat attendu. Toutefois, il ne faudrait pas en déduire qu'il en est ainsi dans tous les cas. En effet, le spectre étudié, qui correspond à un composé cristallisé, est de bonne qualité. Ce n'est pas toujours le cas pour les spectres intéressants. De plus, d'autres phénomènes peuvent venir perturber l'analyse, comme l'émergence d'un autre seuil qui impose de réduire l'étendue du domaine d'analyse, ou une convolution du spectre EXELFS attendu par le spectre des pertes par excitation de plasmon lorsque celles-ci sont importantes. Signalons pour finir que la distribution radiale finale obtenue au cours de cette étude est en tout point similaire à celle résultant de l'analyse d'un spectre EXAFS du même seuil rapportée dans la liltérature [10].

\section{Application des techniques de type EXAFS à la tribologie}

\subsection{Amorphisalion induite par frottement, étudiée par EXELFS}

Dans celte première étude, on s'intéresse au désordre structural induit par frottement de deux surfaces de carbure de silicium sous ultra-vide (frottement sec). Elle porte sur l'analyse EXELFS des structures fines suivant le seuil $L_{23}$ du silicium dans les débris d'usure [11]. Les mesures ont été obtenues dans un microscope électronique à transmission. Figure 5a qui correspond à du $\mathrm{SiC}$ cristallisé sert de témoin tandis que Fig. $5 \mathrm{~b}$ donne la distribution radiale des particules d'usure. La disparition de la contribution de la quatrième couche atomique à $6 \AA$ montre clairement que la taille ultime des grains cristallisés produits par le frottement doit être inférieure à $10 \AA$. Une étude plus détaillée dans un cas similaire (fibres de $\mathrm{SiC}$ amorphe) indiquait un diamètre moyen de cluster de $6 \AA$. Le désordre à l'échelle atomique observé dans les particules d'usures est probablement à l'origine des coefficients de frottement relativement faibles qui ont été observés sous ultra-vide sur ce type de céramiques, bien que les forces d'auto-adhésion soient élevées.

\subsection{Composition chimique et géométrie de la première couche de coordination étudiée par EXAFS}

Dans ce deuxième exemple d'application on s'intéresse aux particules d'usure produites par le frottement de deux matériaux ferreux (acier et fonte) lubrifiés 


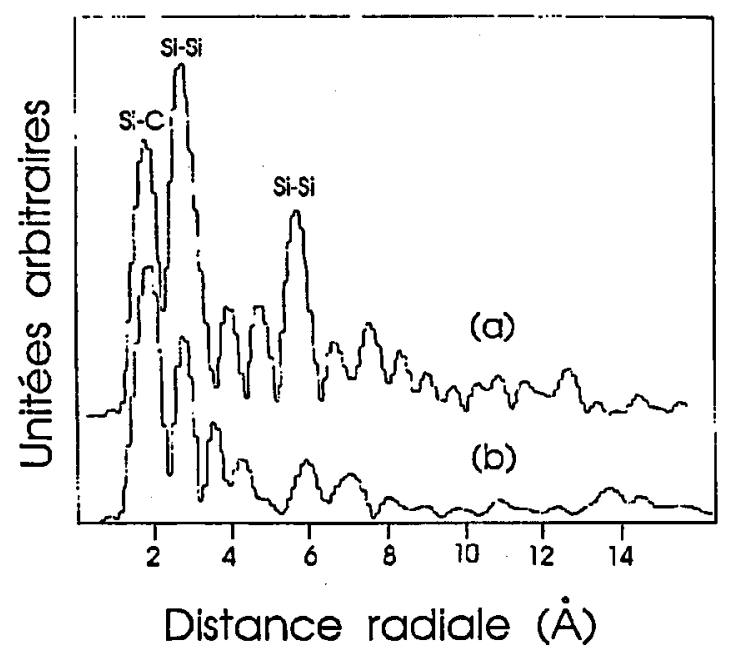

Fig. 5. Distribution radiale provenant de l'analyse EXELFS du seuil $L_{23}$ du silicium (a) dans un cristal de $\alpha$-SiC, (b) dans des particules d'usure produites par frottement sec sous ultra-vide de céramiques industrielles constituées de $\mathrm{SiC}$.

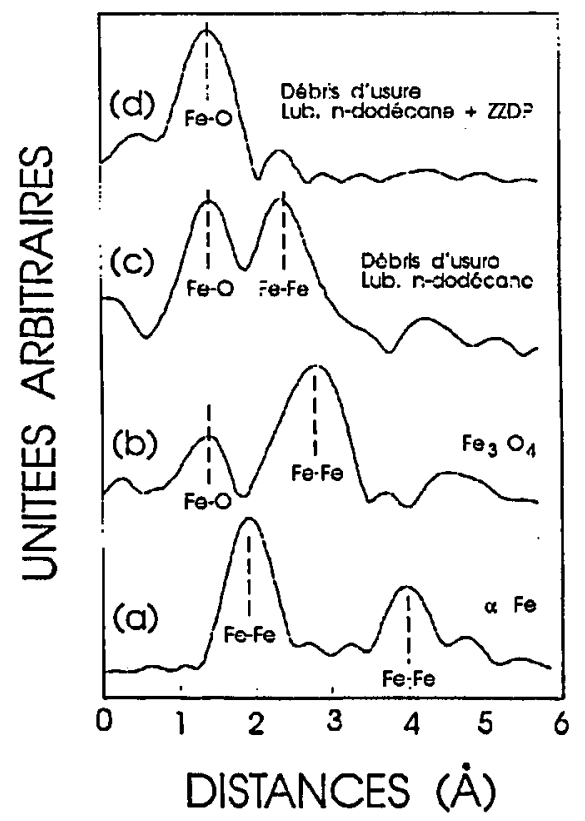

Fig. 6. Distribution radiale provenant de l'analyse EXAFS du seuil $K$ du fer dans différents composés: (a) $\alpha$-Fe cristallisé, (b) $\mathrm{Fe}_{3} \mathrm{O}_{4}$, (c) débris d'usure produits par le frottement de composés ferreux (acier et fonte) lubrifiés par du $n$-dodécane, (d) débris d'usure produits par le frottement de composés ferreux (acier et fonte) lubrifiés a vec une solution à $1 \%$ de ZDDP dans du $n$-dodécane. 
soit à l'aide de $n$-dodécane pur, soit à l'aide d'une solution à $1 \%$ en poids de zinc diiso-propyl-dithiophosphate (ZDDP) dans du $n$-dodécane. La technique d'investigation choisie est l'EXAFS et on étudie le seuil $K$ du fer [12]. Figures $6 \mathrm{a}$ et $6 \mathrm{~b}$ donnent respectivement les distributions radiales de deux échantillons témoins de fer $\alpha$ cristallisé et de $\mathrm{Fe}_{3} \mathrm{O}_{4}$. On constate sur la première que les deux premières couches de coordination du fer sont bien séparées. En ajoutant une correction de déphasage de $0.47 \AA$ aux positions des pics, on retrouve d'ailleurs bien les distances $\mathrm{Fe}-\mathrm{Fe}$ théoriques. La seconde distribution radiale présentée permet de bien distinguer les contributions des liaisons $\mathrm{Fe}-\mathrm{O}$ et $\mathrm{Fe}-\mathrm{Fe}$ de $\mathrm{Fe}_{3} \mathrm{O}_{4}$. Figure $6 \mathrm{c}$ correspond aux particules d'usure obtenues après lubrification par le $n$-dodécane seul. Elle ressemble à celle associée à $\mathrm{Fe}_{3} \mathrm{O}_{4}$, ce qui indique une production importante de cet oxyde au cours du froltement. Si l'on s'intéresse maintenant au cas de la distribution radiale obtenue en présence de ZDDP (Fig. 6d), on n'observe plus que la liaison $\mathrm{Fe}-\mathrm{O}$. Ceci indique la création d'un oxyde complètement amorphisé. Cette différence fondamentale dans la nature des particules d'usure produiles permet d'expliquer l'effet anti-usure observé lors de l'adjonction de ZDDP dans le lubrifiant. On trouvera dans Réf. [12] une discussion approfondie de cet aspect.
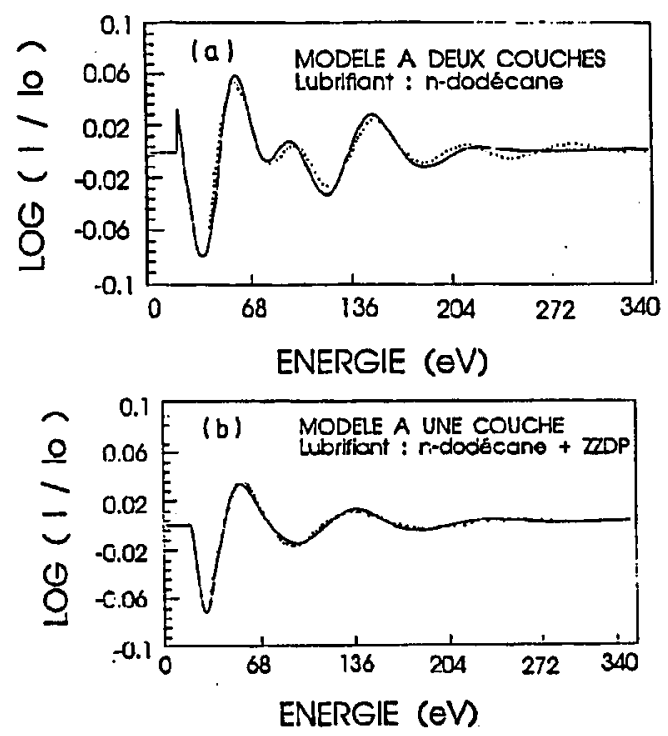

Fig. 7. Comparaison théorie-expérience de spectres EXAFS du seuil $K$ du fer portant sur: (a) les deux premières couches dans des débris d'usure produits par le frottement de composés ferreux (acier et fonte) lubrifiés par du $n$-dodécane, (b) la première couche dans des débris d'usure produits par le frottement de composés ferreux (acier et fonte) lubrifiés a vec une solution à $1 \%$ de ZDDP dans du $n$-dodécane. Traits pleins: expérience; traits pointillés: théorie. 
TABLEAU

Paramètres caractéristiques d'un émetteur Fe dans le composé de référence (poudre de $\mathrm{Fe}_{3} \mathrm{O}_{4}$ ) utilisés pour l'étude des débris d'usure et paramètres optimaux obtenus pour ces débris.

\begin{tabular}{c|c|c|c}
\hline \hline Composé & Voisins & $R[\AA] \pm 0.02$ & $N \pm 0.5$ \\
\hline $\mathrm{Fe}_{3} \mathrm{O}_{4}$ & $\mathrm{O}$ & 1.86 & 5.30 \\
\cline { 2 - 4 } & $\mathrm{Fe}$ & 3.10 & 10.0 \\
\hline $\begin{array}{c}\text { débris d'usure } \\
\text { lub. } n \text {-dodécane }\end{array}$ & $\mathrm{O}$ & 1.82 & 4.06 \\
\cline { 2 - 4 } & $\mathrm{Fe}$ & 3.04 & 8.08 \\
\hline $\begin{array}{c}\text { débris d'usure } \\
\text { lub. } n \text {-dodécane + ZDDP }\end{array}$ & $\mathrm{O}$ & 1.89 & 5.90
\end{tabular}

On peut aller plus loin dans l'analyse quantitative de ces spectres EXAFS. La technique est la suivante: par fenêtrage on isole la contribution d'une couche dans la distribution radiale pour l'échantillon étudié et pour un échantillon témoin de structure la plus voisine possible, et on en prend les TF inverses. Bien entendu, on suppose connus distances et nombres de coordination dans l'échantillon témoin. Cela permet alors de déterminer les fonctions phase et amplitude de rétrodiffusion atomique pour un émetteur situé dans cet échantillon. On utilise ensuite ces fonctions pour calculer la contribution de la couche considérée dans le spectre étudié à l'aide de la formule fondamentale de l'EXAFS, en prenant celle fois distances et nombres de coordination comme inconnues. Ce calcul a été réalisé pour les deux promières couches de Fig. $6 \mathrm{c}$ et la première couche de Fig. $6 \mathrm{~d}$. Le meilleur accord théorie-expérience est représenté sur Fig. 7 tandis que les paramètres optimaux qui conduisent à ce accord sont indiqués dans Tableau. Lorsque le lubrifiant est le $n$-dodécane pur, ces calculs confirment la production de $\mathrm{Fe}_{3} \mathrm{O}_{4}$ (bien que les coordinations obtenues soient un peu plus faibles dans ce travail). Par contre, en présence de.l'additif ZDDP la valeur 5.90 obtenue pour la coordination de l'oxygène est certainement entachée d'erreur, car il est peu probable que le transfert des fonctions phase et amplitude de rétrodiffusion atomique de $\mathrm{Fe}_{3} \mathrm{O}_{4}$ au composé produit par frottement soit vraiment justifié.

\subsection{Etude d'un processus d'amorphisalion par XANES}

On s'intéresse ici aux débris d'usure produits par le même couple que dans l'exemple précédent (acier et fonte). Le lubrifiant utilisé est le $n$-dodécane con- tenant $1 \%$ de ZDDP. L'étude porte sur la forme et la position des structures précédant le seuil $K$ du fer observées sur des spectres EXAFS [13]. Figure 8 rassemble ces renseignements pour les débris d'usure et un certain nombre d'échantillons témoins: $\mathrm{Fe}^{0}$ cristallisé (cubique centré), $\mathrm{Fe}^{3+}$ tétraédrique dans $\mathrm{NiFe}(\mathrm{NiCr}) \mathrm{O}_{4}$ amorphe, $\mathrm{Fe}^{3+}$ octaédrique dans $\mathrm{ZnFeO}_{4}$ amorphe, $\mathrm{Fe}^{2+}$ dans la sidérite.

On observe un déplacement de la position du seuil et du pic le précédent qui correspond à une transition $1 s-3 d$ du fer. Ce déplacement est relié au degré 


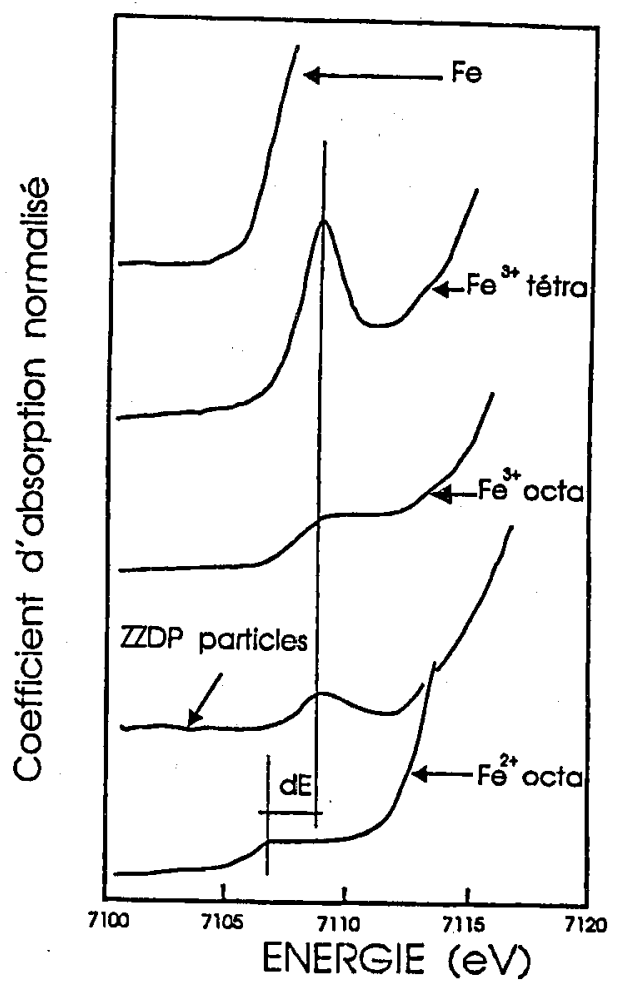

Fig. 8. Analyse XANES du seuil $K$ du fer dans différents composés: $\alpha$-Fe cristallisé, $\mathrm{NiFe}(\mathrm{NiCr}) \mathrm{O}_{4}$ amorphe ( $\mathrm{Fe}^{3+}$ tétraédrique), $\mathrm{ZnFeO}_{4}$ amorphe ( $\mathrm{Fe}^{3+}$ octaédrique), particules d'usure produites par le frottement de composés ferreux (acier et fonte) lubrifiés a vec une solution à $1 \%$ de ZDDP dans du $n$-dodécane, sidérite ( $\mathrm{Fe}^{2+}$ octaédrique).

d'oxydation du fer. Il est difficile à mesurer avec précision en ce qui concerne le seuil lui-même qui est relativement étalé. Par contre, Fig. 8 montre clairement que la position du pic précédent le seuil correspond à un degré d'oxydation $\mathrm{Fe}^{3+}$. L'analyse complète du spectre EXAFS montre que l'on a affaire à un oxyde de fer, ce qui était prévisible.

L'amplitude du pic précédent le seuil est reliée à la symétrie de l'environnement du fer. Là encore, la simple observation de Fig. 8 laisse présager une symétrie octaédrique plutôt que tétraédrique. Toutefois, il faudrait analyser les effets liés à la distorsion du site à l'aide de standards appropriés pour affiner cette conclusion.

L'adjonction de ZDDP dans le lubrifiant a pour principal effet de réduire considérablement l'usure des matériaux frottant au cours du temps. Le film de surface contient évidemment des éléments provenant des molécules de ZDDP (phospore, soufre, zinc et oxygène) qui donnent naissance à des chaînes phosphatées (poly ou pyrophosphates) par des processus thermiques. Les résultats précédents suggèrent 
que ces molécules sont capables de digérer les oxydes de fer produits par le froltement, expliquant ainsi les propriétés anti-usure de cet additif.

\section{Conclusion}

Au cours de ce travail nous nous sommes attachés à montrer l'intérêt des méthodes de type EXAFS pour étudier l'ordre local à l'échelle atomique, sans en cacher les nombreuses limitations. En particulier, Sec. 2.2 fait le point sur les nombreuses difficultés que l'on doit surmonter pour obtenir des distributions radiales fiables. Il s'en dégage une méthodologie permettant de garantir la fiabilité des traitements numériques retenus dans chaque cas particulier et la précision des résultats auxquels ils aboutissent. D'ailleurs, compte tenu de la sensibilité des résultats à ces traitements, ceux-ci ne devraient pas être publiés sans être assortis de l'indication de toutes les méthodes de traitement utilisées et du jeu complet des paramètres adopté. Section 3, qui n'est qu'un simple survol de quelques applications des techniques de type EXAFS à la tribologie, montre de façon irréfutable tout l'intérêt qu'elles présentent dans ce domaine. En fait, dans la plupart des cas, il n'existe pas d'alternative pour atteindre les informations visées. Il conviendrait maintenant de rendre ces techniques plus quantitatives en tentant dans des cas favorables des comparaisons directes théorie-expérience ou en multipliant l'analyse de composés modèles de structure connue susceptibles de servir de base pour la compréhension et l'analyse de nouveaux spectres.

\section{Références}

[1] E.A. Stern, Phys. Rev. B 10, 3027 (1974).

[2] E.A. Stern, D.E. Sayers, F.W. Lytle, Phys. Rev. B 11, 4836 (1975).

[3] K. IIeinz, K. Müller, in: Structural Studies of Surfaces, Springer Tracls in Modern Physice, Vol. 91, Ed. G. Höhler, Springer, Berlin 1982, p. 1.

[1] J.C. Le Bossé, J. Lopez, J. Rousseau, I. Zasada, J. Phys. C, Solid Slate Phys. 21, 565 (1988).

[5] F. Mila, C. Noguera, J. Phys C, Solid State Phys. 20, 3863 (1987).

[6] D.E. Sayers, E.A. Stern, F.W. Lytle, Phys. Rev. Lett. 27, 1204 (1971).

[7] P.A. Lee, G. Beni, Phys. Rev. B 15, 2862 (1977).

[8] J. Lopez, J. Rousseau, J.C. Le Bossé, M. Rjeb, J. Phys., Condens. Matter 3, 5693 (1991).

[9] J.A. Victoreen, J. Appl. Phys. 19, 855 (1948).

[10] P. Lagarde, A.M. Frank, J. Phys. (France) 47, 1389 (1986).

[11] J.M. Martin, T. Le Mogne, Surf. Coat. Technol. 49, 427 (1991).

[12] J.M. Martin, M. Belin, J.L. Mansot, H. Dexpert, P. Lagarde, ASLE Trans. 29, 523 (1986).

[13] M. Belin, J.M. Martin, J.L. Mansot, Tribology Trans. 32, 410 (1989). 